CLINICAL STUDY

\title{
Lipoatrophy in GH deficient patients treated with a long-acting pegylated GH
}

\author{
Philippe Touraine, Gwyn A D'Souza ${ }^{1}$, Ione Kourides ${ }^{2}$, Roger Abs ${ }^{3}$, Paul Barclay ${ }^{2}$, Rujia Xie ${ }^{1}$, Antonio Pico ${ }^{4}$, \\ Elena Torres-Vela ${ }^{5}$, Bertil Ekman ${ }^{6}$ on behalf of the GH Lipoatrophy Study Group \\ Department of Endocrinology and Reproductive Medicine, GH Pitié Salpêtrière, Centre de Référence des Maladies Endocriniennes Rares de la Croissance, \\ Université Paris VI Pierre et Marie Curie, 47-83, Boulevard de l'Hôpital, 75651 Paris Cedex 13, France, ${ }^{1}$ Pfizer Limited, Sandwich, Kent, UK, ${ }^{2}$ Pfizer, \\ New York, New York, USA, ${ }^{3}$ Department of Endocrinology, University of Antwerp, Antwerp, Belgium, ${ }^{4}$ Endocrinology and Nutrition, Hospital General \\ Universitario de Alicante, Universidad Miguel Hernández, Alicante, Spain, ${ }^{5}$ Department of Internal Medicine I, University of Granada Hospital, Granada, \\ Spain and ${ }^{6}$ Division of Internal Medicine, Department of Medical and Health Sciences, Linköping University, Linköping, Sweden
}

(Correspondence should be addressed to P Touraine; Email: philippe.touraine@psl.aphp.fr)

\begin{abstract}
Objective: Changes observed during adult GH deficiency (GHD) are most often reversed with the administration of recombinant human GH (rhGH). To avoid daily injections, a long-acting GH molecule has been obtained by covalent binding of polyethylene glycol (PEG) with rhGH (PEG-GH), allowing weekly s.c. injections. This study was designed to assess its efficacy and safety, in adult GHD subjects.

Design and methods: This was a randomized, double-blind, placebo-controlled, multiple-dose, parallel group study. Subjects were recruited from 34 centers. A total of 105 subjects with GHD were assigned a treatment. They received 6 weekly injections of either PEG-GH or placebo. Subjects were randomized into one out of four treatment groups (Groups A-D) or placebo (Group E). Groups A, B, and C received 1, 3 , and $4 \mathrm{mg}$ PEG-GH respectively, for the first 3 weeks followed by 2, 6, and $8 \mathrm{mg}$ PEG-GH respectively, for the remaining 3 weeks. Group D received $4 \mathrm{mg}$ PEG-GH for 6 weeks. Group E received placebo. The study was suspended because of the development of lipoatrophy in certain subjects and restarted with an injection rotation plan, before being terminated due to further subjects developing lipoatrophy. Results: A total of 13 cases of injection-site lipoatrophy were reported, of which ten were in females and three occurred after the first injection; all cases were independent of PEG-GH dose or IGF1 levels, either basal or under treatment.

Conclusion: The unpredictable occurrence of injection-site lipoatrophy with weekly long-acting pegylated GH molecules may be a limiting factor for their development.
\end{abstract}

European Journal of Endocrinology 161 533-540

\section{Introduction}

Adequate GH secretion is an important factor for appropriate growth in children and for maintaining normal body composition, anabolism, and lipid metabolism in adults. There is strong evidence that adult GH deficiency (GHD) is deleterious in terms of altered body composition, osteoporosis, increased cardiovascular risk, and impaired quality of life (1). Replacement of $\mathrm{GH}$ reverses to a large extent these adverse changes $(2,3)$. Present recombinant human GH (rhGH) preparations have a short elimination half-life (2-3 h) and have to be given by daily s.c. injections. Treatment with rhGH may persist over decades making subject compliance problematic because of the inconvenience of daily injections and needle phobia. A long-acting $\mathrm{GH}$ molecule (polyethylene glycol (PEG)-GH) has been obtained by covalent binding of PEG to GH (4). Its major potential advantage is the reduction in s.c. injections compared to conventional GH replacement therapy.

A randomized, double-blind, placebo-controlled, multiple-dose, parallel group study was designed to assess efficacy and safety after multiple weekly s.c. injections in adult male and female GHD subjects. This study was temporarily suspended following the observation of lipoatrophy at the injection site in some subjects. The protocol was subsequently amended to include an injection site rotation plan. After restart, new cases of injection-site lipoatrophy were reported leading to a definitive termination of the study. We present here the clinical case presentation of these subjects and discuss the possible pathogenesis of GH-induced lipoatrophy. 


\section{Subjects and methods}

\section{Subjects}

This was a multicenter (34 sites), randomized, double-blind, placebo-controlled, multiple-dose, parallel group study (Phase II). A total of 120 subjects were planned in the original protocol. To be included, male and female subjects with hypopituitarism of organic origin or with persisting idiopathic GHD of childhood-onset, had to be 25-60 years of age and present with severe GHD as defined by the $\mathrm{GH}$ Research Society guidelines (GH peak during insulin tolerance test $<3 \mu \mathrm{g} / \mathrm{l})$. Subjects were excluded if they had uncontrolled pituitary tumor growth or diabetes mellitus. Subjects had to be on adequate hormone replacement therapy for at least 6 months and to demonstrate adequate cortisol reserve or to be on adequate glucocorticoid replacement therapy. Subjects could be naïve to $\mathrm{GH}$ replacement therapy, but subjects already treated with GH (non-naïve) had to have a minimum of 3 weeks and a maximum of 4 weeks washout prior to start of study.

\section{Description of the dosage form}

PHA-794428 for injection (lyophilized) is presented as a liquid for a s.c. injection. Each vial contains 5 or $20 \mathrm{mg}$ in $1 \mathrm{ml}$ of $10 \mathrm{mM}$ sodium phosphate $(\mathrm{pH} 6.8)$, $40 \mathrm{mg} / \mathrm{ml}$ mannitol and $5 \mathrm{mg} / \mathrm{ml}$ glycine. This dosage form is presented in a $5 \mathrm{ml}$ Type I clear glass vial with a $20 \mathrm{~mm}$ stopper and $20 \mathrm{~mm}$ aluminum overseal. The placebo was the same composition but without any active drug.

\section{Treatment scheme}

Subjects received weekly injections of either the longacting PEG-GH or placebo during a period of 6 weeks (on day 1 , day 8 , day 15 , day 22 , day 29 , and day 36). Subjects were randomized into one of four treatment groups (Groups A-D) or to the placebo group (Group E). Groups A, B, and $\mathrm{C}$ received 1, 3, and $4 \mathrm{mg}$ PEG-GH respectively, for the first 3 weeks (period 1) followed by 2,6 , and $8 \mathrm{mg}$ PEG-GH respectively, for the remaining 3 weeks (period 2). Group D received $4 \mathrm{mg}$ PEG-GH for six weeks, while Group E received placebo. PEG-GH or placebo was administered in a blinded fashion by a qualified nurse initially as a single s.c. injection in the same thigh, except in two centers where injections were given in the abdomen (time 1). The study was suspended due to the report of injection site reactions subsequently confirmed to be lipoatrophy. An injection rotation plan was proposed, in order to avoid lipoatrophy and the study was restarted (time 2). Injections were given as follows: injection 1 in the lateral right thigh; injection 2 in the lateral left thigh; injection 3 in the abdomen right lower quadrant; injection 4 in the left lower quadrant; injection 5 in the medial right thigh; and injection 6 in the medial left thigh.

\section{Pharmacokinetics}

Serial blood samples $(2 \mathrm{ml})$ were collected on day 1 , days $2-3$, days $4-6$, day 8 , day 15 , days $16-17$, days 18-20, day 22, day 29, day 36, days 37-38, days 39-41, day 43, and days 46-50 for pharmacokinetics (PK) of PEG-GH. Blood samples were centrifuged and serum was stored at $-70 / 80^{\circ} \mathrm{C}$. PEG-GH in human serum was analyzed by dissociation enhanced lanthanide fluorescence immunoassay method with a lower limit of quantification of $10 \mathrm{ng} / \mathrm{ml}$.

\section{Efficacy parameters}

Plasma insulin-like growth factor 1 (IGF1), IGF binding protein-3 (IGFBP-3), and acid-labile subunit (ALS) were used as efficacy biomarkers for PEG-GH activity. Serial blood samples were collected at predose, each study visit and the follow-up visit for IGF1 ( $2 \mathrm{ml}$; same days as PK time points), and blood samples were collected at predose, visit nine (after fourth injection) and visit 14 (1 week after last injection) for IGFBP-3 and ALS (4 ml). Blood samples were centrifuged and the aliquot of sera were stored at $-70 / 80{ }^{\circ} \mathrm{C}$. IGF1 and IGFBP-3 were analyzed by a validated automated chemiluminescent assay system (IMMULITE). ALS samples were analyzed using the ALS immunofluorometric assay. A blood sample $(2 \mathrm{ml})$ was collected twice during follow-up to measure anti-GH antibodies (unit=endpoint titer).

\section{Results}

\section{Subjects}

A total of 157 subjects were screened, 76 at time 1 and 81 at time 2, and 105 subjects were assigned to treatment. A total of 98 subjects completed period 1 (injections 1-3), and 51 completed period 2 (injections 4-6). All subjects were assessed for safety. The baseline characteristics, age, height, weight, and male-to-female ratio, were similar for all treatment groups. The majority $(97 \%)$ of subjects were Caucasian with a mean age of 45.6 years (25-64 years); the mean height was $167.9 \mathrm{~cm}(145.0-194.0 \mathrm{~cm})$, and mean body mass index (BMI) was 27.9 (17.6-43.8). All patients had GHD with a GH peak during insulin tolerance test $<3 \mu \mathrm{g} /$ l. Isolated GHD was reported in $36 \%$ and additional pituitary deficiencies were found in $64 \%$ of the subjects. Diabetes insipidus was reported in $12 \%$. The most common drug treatments reported by investigators, prior to the start of the study drug, were levothyroxine (64\%), hydrocortisone (58\%), GH (51\%) 
and steroid hormones (21\%). Doses of prior GH treatment were similar across the different treatment groups, ranging from 0.1 to $1.2 \mathrm{mg} /$ day with an overall mean dose \pm s.D. of $0.45 \pm 0.25 \mathrm{mg} /$ day.

\section{Safety results}

Lipoatrophy In 13 patients, lipoatrophy was noted at the injection site, 10 cases occurring during time 1 and 3 cases during time 2 of the study. Lipoatrophy occurred in 10 women and 3 men. The first five cases which presented during time 1 of the study are reported below.

Case 1. A 58-year-old woman reported an injection site reaction in the right thigh, after the fifth injection. There were no signs of inflammation, erythema, tenderness, or swelling. The diameter of the lesion was 3-4 cm. Ultrasonography confirmed a subcutaneous tissue thickness of 5.7-5.9 $\mathrm{mm}$ at the right thigh versus a normal subcutaneous tissue thickness at the left thigh (9.3-10 mm), consistent with lipoatrophy. No other abnormalities were detected. The lipoatrophy resolved spontaneously 3 months later after withdrawing the treatment.

Case 2. A 26-year-old woman reported an injection site reaction in the right thigh after the fifth injection. The investigator described subcutaneous lipoatrophy at the injection site. There were no signs of inflammation, redness, swelling, or tenderness. The diameter of the lesion was $5 \mathrm{~cm}$ and the depth $\sim 5.4 \mathrm{~mm}$. Ultrasonography confirmed lipoatrophy, $5.7 \mathrm{~mm}$ right thigh versus normal subcutaneous left thigh depth (11.1 mm). No other abnormalities were noted. Lipoatrophy resolved within 2 months.
Case 3. A 31-year-old woman discontinued treatment after the fourth injection because of pain and redness at the injection site, developing 2 days after the injection, and an associated hematoma. The injections were given in the same area. The subject contacted the investigator complaining that her thighs were different in size. On examination, the right thigh (beneath the injection area) was described as having a narrow area, $20 \times 5 \mathrm{~cm}$, deeper than the surrounding area. Magnetic resonance imaging scan confirmed lipoatrophy with no muscle involvement. Almost 3 months after withdrawal of the treatment, the investigator reported that the lipoatrophy had nearly resolved.

Case 4. A 29-year-old woman who developed an area of lipoatrophy $30 \times 10 \mathrm{~cm}, 2 \mathrm{~cm}$ deep, below the injection site after the fifth injection into the thigh (Fig. 1 left). The injections were delivered into the same area of the thigh. There was no local skin reaction, tenderness, or pain. Ultrasound of the thigh confirmed lipoatrophy, with no muscle involvement. Four months later, it was reported that the lipoatrophy had nearly resolved (Fig. 1 right).

Case 5. A 54-year-old woman who noted a shallow depression in her right thigh after receiving the fifth injection. The injections were delivered into the same area of the thigh. On examination, a $12 \times 4 \times 1 \mathrm{~cm}$ (depth) area of lipoatrophy was detected $3-4 \mathrm{~cm}$ proximal of the injection site on the lateral aspect of the right thigh. There was no pain, tenderness, or discoloration.

Based on these five cases of lipoatrophy, it was decided to suspend the study temporarily; however, after the suspension of the study, further injection site reactions were reported in five patients, from two different centers.
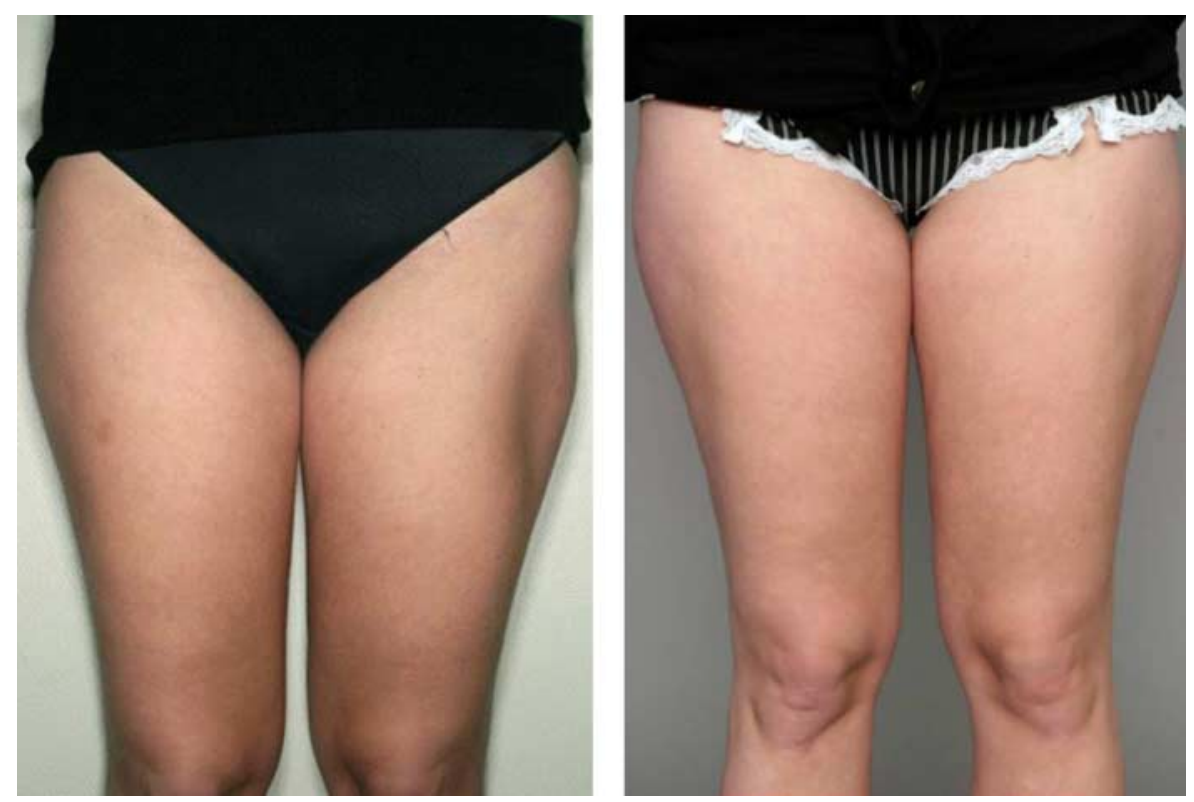

Figure 1 Evolution of the lipoatrophy of the left thigh in subject 4: after the fifth injection of PEG-GH (left) and 4 months after withdrawal of the treatment (right). Full colour version of this figure is available via http://dx. doi.org/10.1530/EJE-09-0422. 
Three of them were reported as presenting lipoatrophy associated with a non-inflammatory adjacent lipohypertrophy (Table 1). It has to be noted that these three patients from two different centers had received their injections in the abdomen and not in the thigh. Since there was no specific pattern in PEG-GH PK and IGF1 levels in these patients, either basal or under treatment, the protocol was amended to include an injection site rotation plan. Nevertheless, three new cases were reported. In these patients, lipoatrophy appeared about four weeks after the first injection. The lipoatrophy was similar to that described in the previous ten cases, with large atrophic lesions, associated with mild pain. All lipoatrophy lesions resolved with time, typically within 8-12 weeks.

At this time the study was permanently discontinued.

Other adverse events Headache was the most frequently reported adverse events (AEs) across all periods and doses. Injection site pain was the most frequently reported treatment-related $\mathrm{AE}$. At least one injection site pain $\mathrm{AE}$ occurred for all doses except for placebo. The majority of AEs were mild to moderate in severity; seven serious AEs occurred, of which three were considered treatment-related. The highest incidence of AEs occurred in Group C-period $2(8 \mathrm{mg}(n=30))$ followed by Group B-period 1 (3 mg $(n=28))$. The highest number of treatment-related AEs occurred in Group A-period $2(2 \mathrm{mg}(n=20)$. The incidence of AEs did not increase with increasing dose of PEG-GH. There were no deaths, dose reductions, or temporary discontinuations due to AEs during the study.

Pharmacokinetics and pharmacodynamics $\mathrm{PEG}-\mathrm{GH}$ trough concentrations were similar in periods 1 and 2 for all groups except Group C (4-8 mg). PEG-GH trough concentrations between male and female subjects or naïve and non-naïve subjects were similar (data not shown). Also, no statistical difference was noted between lipoatrophic and normal subjects (Fig. 2).

The IGF1 concentrations after PEG-GH administration increased over time and IGF1 response increased with dose, from period 1 to period 2 for all treatment groups except Group D (4 mg administered in both periods) where IGF1 concentrations were similar during both periods. No statistical difference was noted between lipoatrophic and normal subjects (Fig. 3). Baseline treatment status did not affect IGF1 concentrations post PEG-GH administration; similar IGF1 levels within each treatment group were observed for naïve and non-naïve subjects (data not shown).

Mean IGFBP-3 concentrations were similar in periods 1 and 2 for all treatment groups; however, no dose response was observed except in Group A. There was no difference in IGFBP-3 concentrations between males and females or between naïve and non-naïve subjects (data not shown). Mean ALS concentrations increased

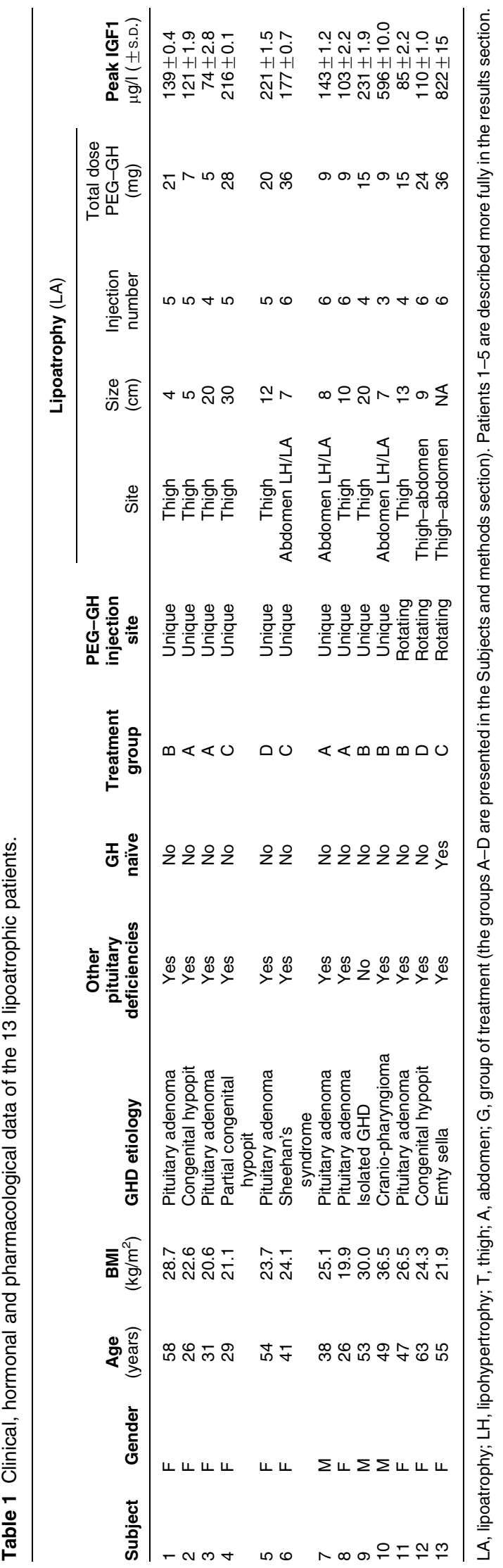


Group A: 1-2mg

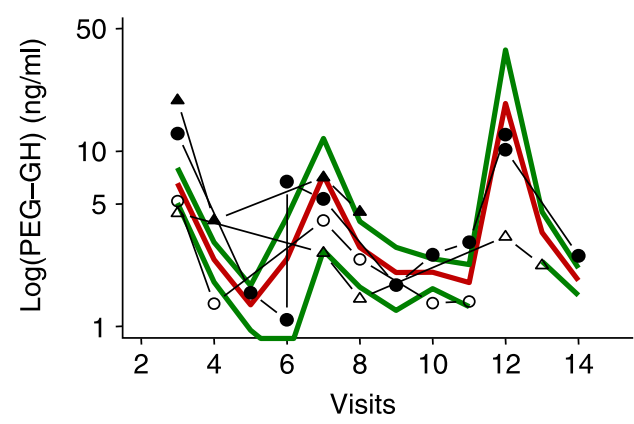

Group C: 4-8mg

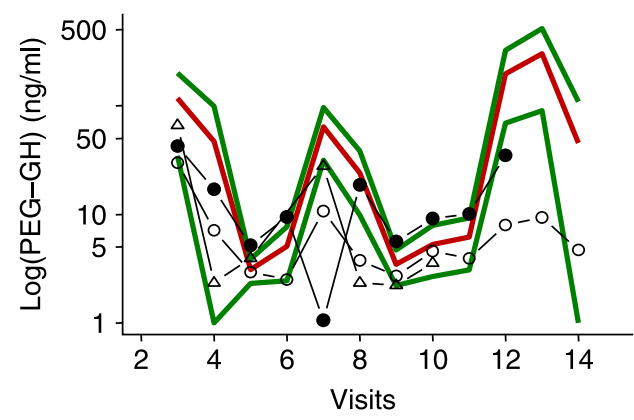

Group B: 3-6 mg

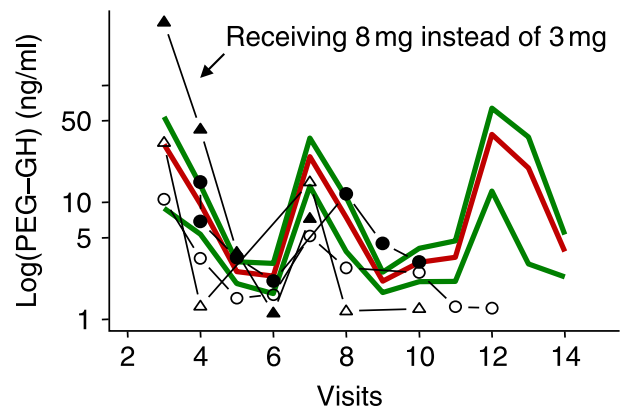

Group D: 4-4mg

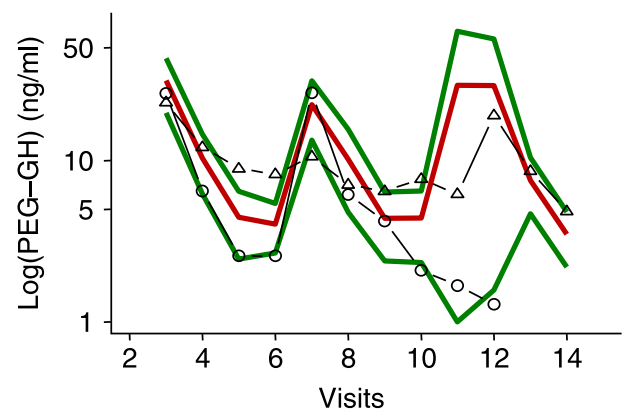

Figure 2 PEG-GH concentration over time profiles for subjects presenting with lipoatrophy: $\triangle, \bigcirc, \bullet, \mathbf{\Delta}$. Mean level and the $90 \% \mathrm{Cl}$ are indicated in red and green respectively. Full colour version of this figure is available via http://dx.doi.org/10.1530/EJE-09-0422.

with increasing PEG-GH dose from period 1 to period 2 for all groups except treatment Group D, where ALS concentrations were similar during both periods (data not shown).

Finally, the majority of subjects were negative for PEG-GH antibodies at baseline (103/105), and during follow-up only two patients developed such antibodies. Although one of them (Patient 7) developed lipoatrophy, the initial titer of the anti PEG-GH antibodies was $<2$ and at endpoint was only 2.03.

\section{Discussion}

This is the first study describing the occurrence of a side effect - injection-site lipoatrophy - in various patients treated with a long-acting, pegylated GH (PEG-GH). In summary, the initial patient reports suggested that lipoatrophy occurred after multiple injections, most often after the fifth or the sixth one; however, during the second part of the study with an injection rotation plan, lipoatrophy also appeared, so 'first dose' occurrence at the injection site cannot be excluded. Indeed, in a single dose study with PEG-GH in children, concomitantly performed with this study, one case of lipoatrophy was observed. The lipoatrophy lesions observed in the present study showed large size (up to $30 \times 10 \mathrm{~cm}$ and $20 \mathrm{~mm}$ depth). Since no skin biopsies were performed, histologic data from these lesions are not available.
Also no predictive factor was clearly identified except female gender; there was no dose effect, and no correlation was found with any other factor such as IGF1 levels, BMI, or previous GH treatment. However, the number of patients studied may have been too low to demonstrate such relationships. The advantage of replacing GHD in adults is now well recognized (5), and several formulations and devices exist for this purpose. A potential limitation to such treatment is the need for daily s.c. injection which can be difficult to maintain in the long run and especially for subjects with needle phobia. The development of long-acting GH molecules permitting a decrease in the number of injections is thus welcome. PEG-GH is a branched pegylated human GH conjugate that has been developed to enable once weekly administration with similar efficacy to daily injections of rhGH. In this phase II study, according to the first administration schedule, PEG-GH was initially repeatedly injected in the same thigh in order to minimize the variability in the $\mathrm{PK}$ and therefore enable exploration of $\mathrm{PK} /$ pharmacodynamic relationships after single and multiple doses. After the report of five cases of lipoatrophy, the study was temporarily suspended to investigate the cause; during this time five new cases were reported. The decision was nevertheless made to reinitiate the study, following the implementation of an injection site rotation plan. The subsequent occurrence of three new cases and even one case in a child in another study led to the termination of the study. 
Group A: 1-2mg

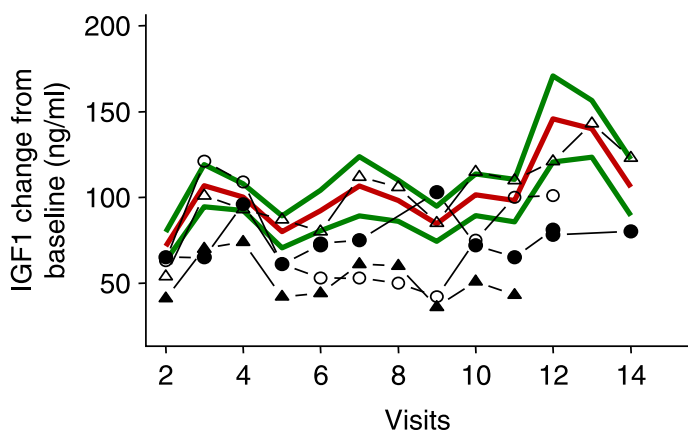

Group C: $4-8 \mathrm{mg}$

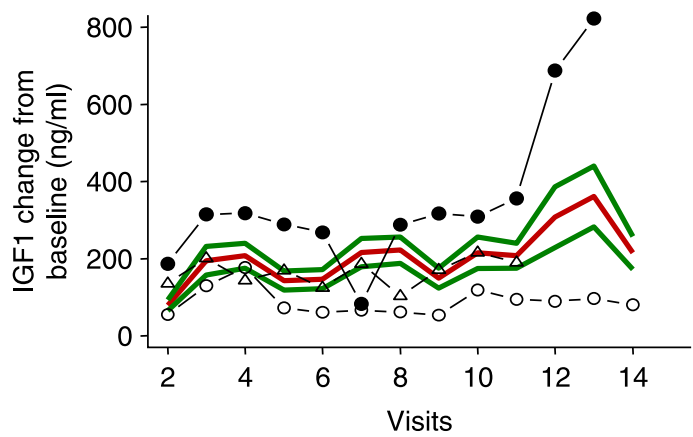

Group B: 3-6mg

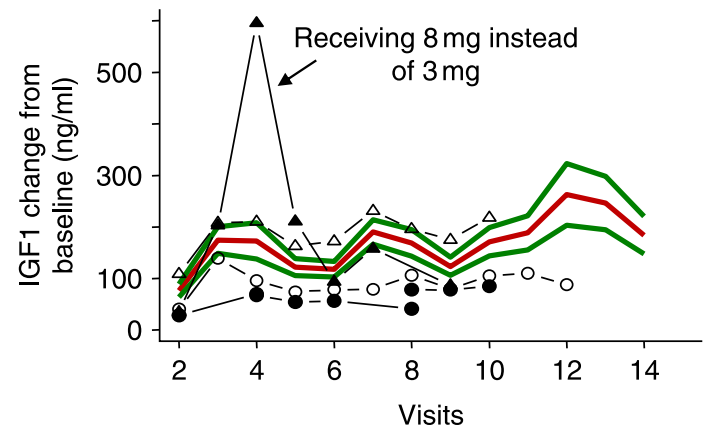

Group D: 4-4 mg

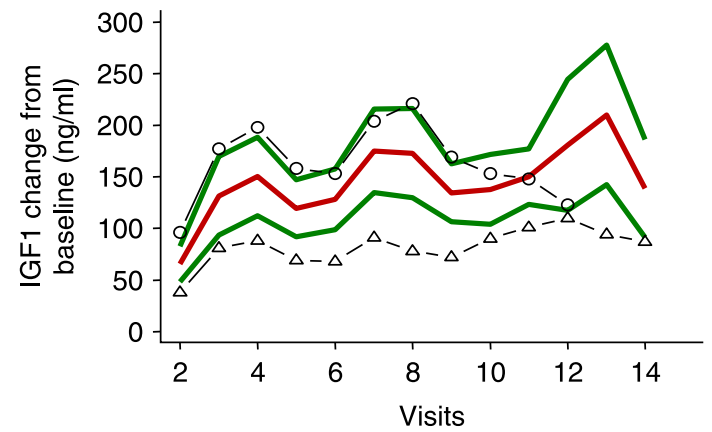

Figure 3 IGF1 concentration over time profiles for subjects presenting with lipoatrophy: $\triangle, \bigcirc, \boldsymbol{O}, \mathbf{\Delta}$. Mean level and the $90 \% \mathrm{Cl}$ are indicated in red and green respectively. Full colour version of this figure is available via http://dx.doi.org/10.1530/EJE-09-0422.

Lipoatrophy refers to a heterogeneous group of syndromes of selective loss of adipose tissue. Acquired lipoatrophy is generally localized and refers to a limited, well-circumscribed subcutaneous depression matching an area of fat loss (6). It can be highly variable in appearance and can range from simple pitting of the skin to large disfiguring disappearance of subcutaneous adipose tissue $(7,8)$. It may be the consequence of needle trauma (9) or result from topically or subcutaneous administered drugs $(6,8,10)$. Lipoatrophy is also more prevalent among females (11). Post-injection lipodystrophy has been reported infrequently in association with a variety of s.c. and intradermally-injected drugs such as insulin, interferon beta, antibiotics, daily GH and GH receptor antagonists (12-14).

With the increased therapeutic use of rhGH since the early 1990s, systemic and local AEs are now well documented and are almost exclusively due to the physiological effect of GH. Very few cases of localized lipoatrophy with daily GH injections have been reported in the literature. Lipoatrophy has been reported to occur after repeated s.c. GH injections at the same site $(15,16)$. In a study of GH treatment of 37 hypopituitary patients with short stature, two females developed lipoatrophic lesions (15). The first one had lipoatrophic lesions at the gluteal injection sites and antibodies against GH. The other one had bilateral areas of lipoatrophy at injections sites, suggesting that extensive areas of fat dissolution around injection sites were due to GH being present subcutaneously long enough to have local lipolytic effects. In another study comparing different routes of $\mathrm{GH}$ administration without site rotation in thirteen children, two developed mild lipoatrophy on the arms (13). Finally, similar AEs have been observed in a cohort of children treated with a long-acting GH formulation with a progressive release of $\mathrm{GH}$ for up to 4 weeks (17). Injection site reactions occurred in nearly all patients and in 13\% lipoatrophy was observed. The lipolytic effect of GH explains why, in the current guidelines, it is recommended to rotate the injection site to minimize this potential complication.

The pathogenesis of lipoatrophy in our cohort of patients is not clear. There was no clinical or biochemical evidence of an inflammatory reaction associated with the injections; there was no significant humoral response as just one of the lipoatrophic patients developed a very low titer of anti-GH or anti-PEG-GH antibodies (Patient 7); there were no issues identified in the preparation or administration technique of the study drug. The fact that PEG-GH has a higher molecular weight than GH may suggest a delayed absorption after s.c. injection. However, the PEG-GH concentrations 
over time profiles for the patients with lipoatrophy were similar to those of the non-lipoatrophy patients, with PEG-GH concentrations within the range of the group. Finally, one patient with very high IGF1 levels (Patient 13) developed lipoatrophy; in this case, a manufacturing assessment was performed and revealed no deviations in the clinical supplies. The dosage and administration instructions from all sites were reviewed, and no significant breech in the instructions was found.

In such a context, although unproven, the most probable hypothesis remains a direct lipolytic effect of $\mathrm{GH}$ on adipose tissue. Recent publications have detailed the direct effects of $\mathrm{GH}$ on adipocytes and adipogenesis. GH stimulates lipolysis in mature adipocytes and primary preadipocytes but promotes adipogenesis in preadipocyte cell lines. Mouse prolactin and mouse $\mathrm{GH}$ stimulate IGF1 mRNA in differentiated cells but not in undifferentiated cells $(18,19)$. GH increases lipolysis as a direct effect of GH on adipocytes, as well as a decrease of lipoprotein lipase activity, diminishing the flow of fatty free acids to the adipocyte. $\mathrm{GH}$ seems to have the potential to reduce the volume of mature adipocytes, thus inhibiting the expansion of adipose tissue and reducing body fat (20). IGF1 per se may also have direct actions on adipocyte diffentiation (21-23); in a recent study, IGF1 signaling has been demonstrated to be decreased in children small for gestational age (SGA) leading, potentially, to the metabolic abnormalities described in these children (24).

Unfortunately, an appropriate non-clinical model to study PEG-GH induced effects on adipose tissue is not available. Skin biopsies would have been helpful in clarifying the nature of the local reaction, but none were performed in the present study. This would have been of particular interest in the three patients presenting combined lipoatrophy and lipohypertrophy, who had abdominal injections, in order to study the histology of these apparently different lesions.

The present study confirms the increased prevalence of lipoatrophy in women compared to men. This gender difference has also been described in HIV patients treated with antiretroviral agents (25) and in acromegalic patients receiving pegvisomant (14) or octreotide (26). The observed female preponderance may be attributed to a larger accumulation of subcutaneous adipose tissue in women, or to a gender-specific adipocyte response.

In conclusion, in this pilot study, the use of PEG-GH has been associated with a risk of developing lipoatrophy at the injection site. This side effect, even if spontaneously resolving, may limit the development of long-acting GH molecules. Further studies are needed to better understand the pathogenesis of these lesions.

\section{Declaration of interest}

P Touraine, R Abs, A Pico, E Torres-Vela and B Ekman have nothing to declare; G A D'Souza, I Kourides, P Barclay and R Xie are employees of Pfizer.

\section{Funding}

This study has been registered in the Clinicaltrials.gov database with the number NCT00308464. It was sponsored by Pfizer.

\section{Acknowledgements}

We specially thank the patients who participated in the PEG-GH study. The other investigators in this study were: M Andersen; P Beck-Peccoz; A Beckers; M A Bex; S R Bornstein; S Cannavo; J Cap; P Chanson; A M Colao; M Faust; I Halperin; M Karbownik-Lewinska; L De Marinis; W M Drake; E M Erfurth; E Ghigo; V Hana; P H Kann; P Laurberg; J P Miell; A Milewicz; J Payer; A M Pereira; G T'Sjoen; J Sowinski; G K Stalla; P J Trainer; and J Wass. The GH Lipoatrophy Study Group includes T Brue, F F Casanueva, P Czernichow, B Delemer, J De Schepper, U Feldt-Rasmussen, J W Gregory, J O L Jørgensen, G Johannsson, L O Kristensen, C Mattsson, M Pura, P Vanuga.

\section{References}

1 Molitch ME, Clemmons DR, Malozowski S, Merriam GR, Shalet SM, Vance ML, Endocrine Society's Clinical Guidelines Subcommittee \& Stephens PA. Evaluation and treatment of adult growth hormone deficiency: an Endocrine Society Clinical Practice Guideline. Journal of Clinical Endocrinology and Metabolism 200691 1621-1634.

2 Bengtsson BA, Edén S, Lönn L, Kvist H, Stokland A, Lindstedt G, Bosaeus I, Tölli J, Sjöström L \& Isaksson OG. Treatment of adults with growth hormone $(\mathrm{GH})$ deficiency with recombinant human GH. Journal of Clinical Endocrinology and Metabolism $1993 \mathbf{7 6}$ 309-317.

3 Verhelst J \& Abs R. Long-term growth hormone replacement therapy in hypopituitary adults. Drugs 200262 2399-2412.

4 Webster R, Xie R, Didier E, Finnessy J, Edgington A \& Walker D. PEGylation of somatropin (recombinant human growth hormone): impact on its clearance in humans. Xenobiotica $2008 \mathbf{3 8}$ 1341-1351.

5 Simpson H, Savine R, Sönksen P, Bengtsson BA, Carlsson L, Christiansen JS, Clemmons D, Cohen P, Hintz R, Ho K, Mullis P, Robinson I, Strasburger C, Tanaka T \& Thorner M. GRS Council. Growth hormone replacement therapy for adults: into the new millennium. Growth Hormone \& IGF Research 200212 1-133.

6 Garg A. Lipodystrophies. American Journal of Medicine 2000108 143-152.

7 Dahl PR, Zalla MJ \& Winkelmann RK. Localized involutional lipoatrophy: a clinicopathologic study of 16 patients. Journal of the American Academy of Dermatology 199635 523-528.

8 Morgan AM. Localized reactions to injected therapeutic materials. Part 1. Medical agents. Journal of Cutaneous Pathology 199522 193-214.

9 Drago F, Rongioletti F, Battifoglio ML \& Rebora A. Localised lipoatrophy after acupuncture. Lancet $1996 \mathbf{3 4 7} 1484$.

10 Yamamoto T, Yokozeki H \& Nishioka K. Localized involutional lipoatrophy: report of six cases. Korean Journal of Dermatology 2002 29 638-643.

11 Gómez-Espejo C, Pérez-Bernal A \& Camacho-Martínez F. A new case of semicircular lipoatrophy associated with repeated external microtraumas and review of the literature. Journal of the European Academy of Dermatology and Venereology $200519459-461$.

12 Kamal AD, Dixon AN \& Bain SC. Safety and side effects of the insulin analogues. Expert Opinion on Drug Safety 20065 131-143.

13 Russo L \& Moore WV. A comparison of subcutaneous and intramuscular administration of human growth hormone in the therapy of growth hormone deficiency. Journal of Clinical Endocrinology and Metabolism 198255 1003-1006.

14 Bonert VS, Kennedy L, Petersenn S, Barkan A, Carmichael J \& Melmed S. Lipodystrophy in patients with acromegaly receiving pegvisomant. Journal of Clinical Endocrinology and Metabolism 2008 93 3515-3518. 
15 Collipp PJ, Thomas J, Curti V, Sharma RK, Maddaiah VT \& Cohn SE. Body composition changes in children receiving human growth hormone. Metabolism 197322 589-595.

16 Underwood LE, Voina SJ \& Van Wyk JJ. Restoration of growth by human growth hormone (Roos) in hypopituitary dwarfs immunized by other human growth hormone preparations: clinical and immunological studies. Journal of Clinical Endocrinology and Metabolism 197438 288-297.

17 Brown LR. Commercial challenges of protein drug delivery. Expert Opinion on Drug Delivery 20052 29-42.

18 Fleenor D, Arumugam R \& Freemark M. Growth hormone and prolactin receptors in adipogenesis: STAT-5 activation, suppressors of cytokine signaling, and regulation of insulin-like growth factor I. Hormone Research $2006 \mathbf{6 6} 101-110$.

19 Vikman K, Isgaard J \& Edén S. Growth hormone regulation of insulin-like growth factor-I mRNA in rat adipose tissue and isolated rat adipocytes. Journal of Endocrinology 1991131 139-145.

20 Takahashi S \& Satozawa N. The 20-kD human growth hormone reduces body fat by increasing lipolysis and decreasing lipoprotein lipase activity. Hormone Research 200258 157-164.

21 Mauras N \& Haymond MW. Are the metabolic effects of GH and IGF-I separable? Growth Hormone \& IGF Research 200515 $19-27$.
22 Blüher S, Kratzsch J \& Kiess W. Insulin-like growth factor I, growth hormone and insulin in white adipose tissue. Best Practice and Research. Clinical Endocrinology and Metabolism 200519 577-587.

23 Holzenberger M, Hamard G, Zaoui R, Leneuve P, Ducos B, Beccavin C, Périn L \& Le Bouc Y. Experimental IGF-I receptor deficiency generates a sexually dimorphic pattern of organ-specific growth deficits in mice, affecting fat tissue in particular. Endocrinology 2001142 4469-4478.

24 Iñiguez G, Ormazabal P, López T, Maldonado D, Avila A, Román R \& Cassorla F. IGF-IR/ERK content and response to IGF-I and insulin in adipocytes from small for gestational age children. Growth Hormone E IGF Research 200919 256-261.

25 Balasubramanyam A, Sekhar RV, Jahoor F, Jones PH \& Pownall HJ. Pathophysiology of dyslipidemia and increased cardiovascular risk in HIV lipodystrophy: a model of 'systemic steatosis'. Current Opinion in Lipidology 200415 59-67.

26 Atmaca A \& Erbas T. Lipoatrophy induced by subcutaneous administration of octreotide in the treatment of acromegaly. Experimental and Clinical Endocrinology and Diabetes 2005113 340-343.

Received 13 July 2009

Accepted 1 August 2009 